\title{
Bidirectional influences of vibrotactile stimuli on modification of the human acoustic startle reflex
}

\author{
TERRY D. BLUMENTHAL \\ Wake Forest University, Winston-Salem, North Carolina \\ and \\ EUGENE A. TOLOMEO \\ Hamilton College, Clinton, New York
}

\begin{abstract}
The present study deals with the effects of a vibrotactile prepulse on the acoustic startle reflex. An $85-\mathrm{dB}(A)$ broadband noise was used to elicit the startle reflex, and the amplitude, latency, and probability of integrated electromyographic activity of the muscle beneath the eye were measured. Experiment 1 involved the presentation of vibrotactile prepulses at 20-dB SL to the hand, at lead times (stimulus onset asynchronies) of 0 to $400 \mathrm{msec}$ before the acoustic startle stimulus. When compared to control (no prepulse) responding, a vibrotactile prepulse with a lead time of 25 msec caused a significant increase in response amplitude, while prepulses at lead times of 150 to $\mathbf{4 0 0} \mathrm{msec}$ inhibited response amplitude. Response latency was significantly reduced (there was facilitation) in comparison with results for the control condition, at lead times of $25,50,75$, 100,200 , and 350 msec. Experiment 2 involved presenting subjects with two prepulses (at lead times of 25 and $200 \mathrm{msec}$ ) on the same trial or on separate trials. A prepulse at a lead time of 25 msec caused an increase in response amplitude, but had no effect on response latency or probability. A prepulse at a lead time of $200 \mathrm{msec}$ caused a decrease in response probability, but had no effect on response amplitude or latency. Response amplitude and probability were larger when both prepulses were presented on the same trial than they were when the 200 -msec lead time prepulse was presented alone. These data support the hypothesis that the mechanisms for inhibition and facilitation of the human startle reflex are partially independent, and that crossmodal modification of the startle response may involve different mechanisms than those responsible for modification within a single sensory system.
\end{abstract}

The purpose of the present study was to investigate the modification of a brainstem reflex by a weak stimulus presented in a sensory modality different from that of the reflex-eliciting stimulus. The startle response, a brainstem reflex in response to a sudden intense stimulus, has been studied in a variety of species, including human beings, and at a range of ages in rats and human beings. The startle-eliciting stimulus can be in any modality: tactile (Hoffman, Cohen, \& Stitt, 1981), auditory (Blumenthal \& Berg, 1986a, 1986b; Graham, 1980), or visual (Stitt, Hoffman, Marsh, \& Schwartz, 1976). The startle reflex can be modified by a suprathreshold change in the sensory environment prior to the presentation of the startleeliciting stimulus. This reflex modification is not dependent on learning, conditioning, or motivation on the part

Portions of this research were conducted by Eugene A. Tolomeo in partial fulfillment of the requirements of Honors in Psychology at Hamilton College. They were presented at the Southeastern Psychological Association Convention, New Orleans, in April 1988. Address reprint requests to Terry D. Blumenthal, Department of Psychology, Wake Forest University, Box 7778 Reynolda Station, Winston-Salem, NC 27109 . of the subject (Hoffman \& Ison, 1980). The modifying prepulse can be in any modality: vibrotactile (Blumenthal \& Gescheider, 1987), electrocutaneous (Graham, 1980), auditory (Graham, 1975; Stitt, Hoffman, \& Marsh, 1976), or visual (Ison \& Hammond, 1971). Modification of the response occurs whether the prepulse and the startle stimulus are in the same or in different modalities (Anthony, 1985; Blumenthal \& Gescheider, 1987; Graham, 1980).

The procedure of reflex modification may have many practical applications, as, for example, in audiometry, or in other types of perceptual assessment that involve individuals, such as infants or handicapped children and adults, who are difficult to test with standard psychophysical methods (Ison et al., 1986; Marsh, Hoffman, \& Stitt, 1978). Sensory thresholds and capabilities could be determined through the use of a range of prepulse intensities, frequencies, durations, and so forth, in a startle modification session. For instance, as prepulse intensity is decreased, the point at which startle modification no longer occurs can be regarded as a threshold for detection of the prepulse, regardless of modality. If the prepulse modifies the startle reflex, then the sensory system must 
be functioning to some extent, at least up to the level of the midbrain, where the modification of startle is believed to occur (Leitner, Powers, \& Hoffman, 1979).

A prepulse can result in reflex facilitation or inhibition, depending on the interval between the prepulse and the startle-eliciting stimulus (this stimulus onset asynchrony is referred to as "lead time"). It is possible to manipulate response amplitude and latency independently of each other, which suggests that they are controlled by separate neural pathways. At a lead time of $30 \mathrm{msec}$, a 70-dB SPL acoustic prepulse causes a facilitation of (decrease in) the latency of the eyeblink component of the human acoustic startle response to a 105-dB SPL eliciting stimulus, and an inhibition of (decrease in) blink magnitude. At a lead time of $120 \mathrm{msec}$, the acoustic prepulse has no significant effect on response latency, but inhibition of blink amplitude is still significant (Graham \& Murray, 1977). Blumenthal and Verma (1988) also found that a 70-dB SPL acoustic prepulse at a lead time of $50 \mathrm{msec}$ caused a reduction of the amplitude of the human acoustic startle response to a 95-dB SPL eliciting stimulus, but response latency was not affected at this lead time. ${ }^{1}$ Ison et al. (1986) have shown that an electrical prepulse inhibits the amplitude of the electrically elicited startle response in humans beings, at lead times of $50 \mathrm{msec}$ or greater. Also, Hoffman, Cohen, and English (1985) have shown that a tactile prepulse (glabellar tap) inhibits a tactile startle stimulus (glabellar tap) at a lead time of 200 msec.

If the prepulse and startle stimulus are in different modalities, reflex modification varies as a function of prepulse lead time. Graham (1980) found that, with either a visual or an electrocutaneous prepulse at a short lead time ( $<100 \mathrm{msec})$, there was facilitation of both the magnitude and the latency of the human acoustic startle reflex. At longer lead times ( $>120 \mathrm{msec}$ ), there was inhibition of reflex magnitude but no change in reflex latency. ${ }^{2}$ Reiter and Ison (1977) found that a visual prepulse caused a slight facilitation of tactile blink amplitude (elicited by an airpuff to the side of the face). Blumenthal and Gescheider (1987), using 20-dB SL vibrotactile prepulses, found facilitation of the human acoustic startle response (increased amplitude and decreased latency) at a 50-msec lead time, and startle inhibition (decreased amplitude and probability, but no effect on latency) for prepulses at a 200-msec lead time, supporting Graham's (1980) findings. These data, taken together with the data from the use of acoustic prepulses, show that, at lead times of approximately $100 \mathrm{msec}$ or more, the prepulses in all modalities tested have a similar effect on the human acoustic startle response (inhibition of response amplitude and no effect on response latency). However, at shorter lead times, differences between prepulse modalities are found. Prepulses in all modalities cause decreased response latency, but response amplitude is inhibited by acoustic prepulses and facilitated by visual, electrocutaneous, and vibrotactile prepulses. This suggests that, at short lead times, response amplitude inhibition occurs if the prepulse and startle stimulus are in the same modality, and that facilitation occurs if they are in different modalities. It is clear that more research is necessary for these crossmodal priming effects to be explained.

Using acoustic prepulses and measuring acoustic startle in rats, Stitt, Hoffman, and Marsh (1976) found that the response amplitude inhibition produced by a $64-\mathrm{msec}$ lead time prepulse was not influenced by the presence of a 4-msec lead time prepulse. Also, the latency facilitation produced by the 4-msec lead time prepulse was not affected by the presence of the 64-msec lead time prepulse. They concluded that the independence of the modification effects of the two prepulses suggests that amplitude inhibition and latency facilitation are mediated by separate neural mechanisms. A lesion in the area of the lateral tegmentum of the rat midbrain extinguishes the amplitude inhibition caused by a prepulse with a lead time of $50 \mathrm{msec}$, but it has no effect on the latency facilitation caused by a prepulse at a 4-msec lead time (Leitner et al., 1979). Also, the neural systems responsible for facilitation of startle in the rat have been found to mature earlier than those responsible for startle inhibition (Gallager, Kehne, Wakeman, \& Davis, 1983). Ornitz, Guthrie, Kaplan, Lane, and Norman (1986) provide data that suggest that these independent mechanisms mature at different rates in the human infant as well. Graham and Murray (1977) postulate that reflex amplitude inhibition may be due to the activity of a "transient" system, while reflex latency facilitation is due to the activity of a "sustained" system, which primes the startle pathway. These transient and sustained systems are based on short-time-constant and long-time-constant neurons, which have been identified at all levels of the auditory pathway in cats and rats by Gersuni (1971). These neurons may form the basis for a physiological mechanism that accounts for the differences in prepulse effects shown by modification of response amplitude and response latency. Given our knowledge of the physiology of reflex modification in subhuman species, this research can support inferences concerning the mechanisms underlying human stimulus processing.

Given the findings of Blumenthal and Gescheider (1987) and Graham (1980), it was predicted in the present study that a vibrotactile prepulse would cause acoustic startle reflex facilitation at short lead times, shown by both decreased latency and increased amplitude. At longer lead times, the inhibition of response amplitude was predicted, with no significant effect on response latency. These predictions were tested in Experiment 1. On the basis of the results, a second experiment, analogous to that of Stitt, Hoffman, and Marsh (1976), was performed. In Experiment 2, human subjects were presented with one or two prepulses on each trial. One of the prepulses had a lead time that normally would cause facilitation of the startle reflex; the other prepulse had a lead time that normally would cause inhibition of the startle reflex. For the twoprepulse condition, it was predicted that there would be a compensation of the facilitatory effects of one prepulse 
by the inhibitory effects of the other prepulse, resulting in an attenuation of the effect found with each prepulse separately.

\section{EXPERIMENT 1}

\section{Method}

Subjects. Fourteen college undergraduates ( 6 males and 8 females), with an average age of 20 years and 2 months (range = 18 years, 5 months to 21 years, 9 months), participated in Experiment 1 . All of the students were in good health and reported no history of hearing loss.

Apparatus. The vibrotactile stimuli were produced by a Ling 203 Shaker (vibrator) attached to a $3.0-\mathrm{cm}^{2}$ contoured circular contactor, which protruded through a hole in a rigid table next to the subject's chair in the testing booth (in order to restrict vibration to the immediate vicinity of the vibrator). The stimulus that activated the vibrator was produced by a Hewlett-Packard $200 \mathrm{AB}$ audio oscillator, gated through a Coulbourn electronic switch and a Daven step attenuator, followed by a Grason-Stadler E2362A continuous recording attenuator. The signal was then amplified by a Crown D60 stereo amplifier and presented to the vibrator. The vibrator's movement was monitored with an Endevco 2221D accelerometer and an Endevco charge amplifier; a Keithley 179A voltmeter was used to measure the accelerometer output. The sound made by the vibrator was masked by a $40-\mathrm{dB}(\mathrm{A})$ noise stimulus delivered continuously by an EPI 1200 loudspeaker. This noise was produced by a Grason-Stadler $901 \mathrm{~B}$ white-noise generator, which was filtered through an Allison Labs 24ABR filter set at a high-frequency cutoff of $75 \mathrm{~Hz}$.

The acoustic startle stimuli were produced by a Coulbourn noise generator, gated through a Coulbourn electronic switch, and amplified by a Crown D60 stereo amplifier, the output of which was presented to the subject by a second EPI 1200 loudspeaker. A General Radio 1551C sound level meter was used to check the sound intensity. A Hewlett-Packard $400 \mathrm{D}$ voltmeter was used to check the voltages applied to the speakers.

The eyeblink component of the startle reflex was measured with Beckman miniature biopotential electrodes, which detected the electromyographic (EMG) activity of the periorbital flexor muscle around the eye (orbicularis oculi). A Coulbourn high-gain bioamplifier/coupler, with filters passing $90-250 \mathrm{~Hz}$, was used to amplify the EMG signal. A Coulbourn contour-following integrator (with a time constant of $80 \mathrm{msec}$ ) rectified and integrated the output. The output of the bioamplifier was also displayed on-line by a Tektronix 5111 storage oscilloscope. The integrated EMG was digitally sampled every $2 \mathrm{msec}$ for $500 \mathrm{msec}$ after the onset of the vibrotactile prepulse, by an Apple II+ microcomputer (12-bit accuracy). In the control condition, the computer started sampling at the onset of the startle pulse. These samples were then displayed on the monitor screen and stored on the computer disk.

Procedure. The area under the subject's left eye was cleansed with a cotton swab soaked in alcohol, and two electrodes were then placed below the eye, as close to the bottom of the eyeball as possible without impairing the subject's eye movement. One of the electrodes was several millimeters below the pupil, and the other was immediately temporal to and slightly higher than the first. A ground electrode was placed on the subject's left forearm. The subject was then seated in the testing booth and asked to look at the stereo speaker (to minimize extraneous eye movements). The subject placed the thenar eminence of the right hand on the vibrator disk. The experimenter explained that the right arm could rest on the table through which the disk protruded, but that the hand was not to be moved at any time during the session.

The startle stimulus was an $85-\mathrm{dB}(\mathrm{A})$ SPL broadband noise $(20 \mathrm{~Hz}-20 \mathrm{kHz})$, with a $50-\mathrm{msec}$ duration and a linear rise/fall time of $1 \mathrm{msec}$ delivered from a loudspeaker located $1.5 \mathrm{~m}$ in front of the subject. The vibrotactile prepulse was a $75-\mathrm{Hz}$ vibration delivered to the thenar eminence of the right hand by a vibrating disk. The duration of the vibration, from the beginning of the rise to the beginning of the fall, was $20 \mathrm{msec}$, with a rise/fall time of $5 \mathrm{msec}$. The intensity of vibration was $20 \mathrm{~dB}$ above each individual subject's psychophysical threshold (SL). The intertrial interval averaged $20 \mathrm{sec}$ (range $=15-25 \mathrm{sec}$ ). The prepulse was delivered at a lead time (stimulus onset asynchrony) of $0,25,50,75,100,150$, $200,250,300,350$, or $400 \mathrm{msec}$, with a control condition in which no prepulse was delivered.

The Békésy tracking method was used to determine the vibrotactile threshold (Békésy, 1947). A 20-msec-long, 75-Hz vibrotactile stimulus was presented every $600 \mathrm{msec}$, and the subject controlled the intensity of this stimulus by holding down a switch (decreasing the intensity of the pulses) when the vibration could be felt, and releasing the switch (increasing the intensity of the pulses) when the vibration could not be felt. After oscillation around the threshold level for 1 to $2 \mathrm{~min}$, the threshold reading was taken with the Keithley voltmeter. The vibrotactile stimulus intensity was then increased by $20 \mathrm{~dB}$. Stimuli were presented in eight blocks of 12 trials each, with each trial including one instance of each of the 12 stimulus conditions ( 11 lead time conditions and the control condition). The order of stimulus presentation within each trial block was randomly determined.

The response amplitude was taken as the difference between the level of the integrated EMG at response onset and at the peak of the change in the slope of the integrated EMG. The latency was the time between the onset of the startle pulse and the onset of the response. Only responses beginning between 20 and $100 \mathrm{msec}$ after the startle pulse onset were examined in order to limit analysis to stimulus-elicited blinks. The probability of responding was determined by dividing the number of nonzero trials by the total number of trials. Blumenthal and Berg (1986b) describe response analysis techniques used in this area of research. The data were analyzed using a separate MANOVA (BMDP4V) for each dependent measure (startle amplitude, latency, and probabiltiy), and contrasts within the MANOVA were used to follow up significant effects and to compare each prepulse condition with the control condition. MANOVA was used to guard against violations of the assumption of sphericity, and conservatively corrected degrees of freedom (Greenhouse-Geisser) are reported. To follow up some of the significant main effects within groups, $t$ tests were used. Sex ( 2 levels) was a between-subjects factor, and lead time, or stimulus onset asynchrony (11 levels), was a within-subject factor.

\section{Results}

There was no significant difference between males and females in response amplitude, so the results for the two groups were pooled for subsequent analyses. Response amplitude varied as a function of lead time $[F(4,43)=$ $10.48, p<.0001$; see Figure 1]. At a lead time of $25 \mathrm{msec}$, response amplitude was significantly increased compared with the amplitude in the control condition $[F(1,12)=28.31, p<.001]$. At lead times of 0,5075 , and $100 \mathrm{msec}$, response amplitude did not differ significantly from the amplitude in the control condition. A prepulse caused a significant decrease in response amplitude at lead times of $150,200,250,300,350$, and $400 \mathrm{msec}[F(1,12)=10.24,7.47,7.29,8.69,5.07$, and 6.10 , respectively, $p s<.05]$.

For response latency, a significant sex effect was found $[F(1,12)=12.99, p<.005]$, as was a sex $\times$ lead time interaction $[F(5,65)=2.73, p<.025$; see Figure 2]. 


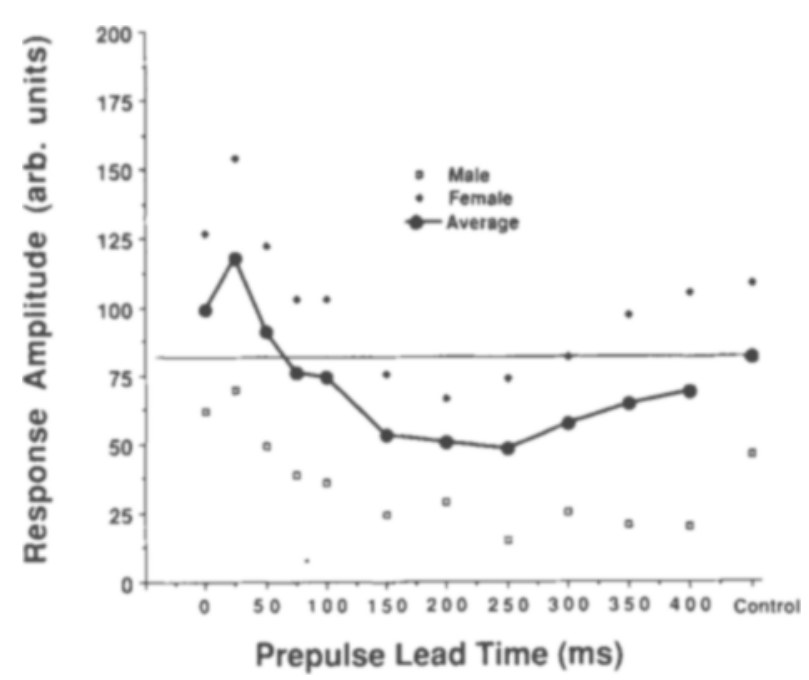

Figure 1. Acoustic startle response amplitude as a function of vibrotactile prepulse lead time.

In the control condition, male response latency was greater than female response latency $[t(12)=3.24, p<.01]$. However, if the analysis was conducted without data from the 400-msec lead time condition, the sex $\times$ lead time interaction was not significant. The difference between the 400-msec lead time prepulse condition and the control condition was significantly different for males and females $[t(12)=3.74, p<.005]$. When data were pooled across sex, latency was significantly reduced compared to the control latency, at lead times of $25,50,75$, 100,200 , and $350 \mathrm{msec}[F(1,12)=5.19,8.36,7.84$, $5.15,6.16$, and 8.05 , respectively, $p s<.05$ ]. There was no significant difference in response latency between the control condition and trials with lead times of $0,150,250$, or $300 \mathrm{msec}$.

There was a significant difference in response probability between males and females $[F(1,12)=8.35$, $p<.025$; see Figure 3]. A lead time effect was also found $[F(4,47)=4.03, p<.01]$. Although the sex $\times$ lead time interaction was not significant, probability tended to decrease more for males than for females as lead time increased. Response probability in the control condition was not different for the two sexes. Only a lead time of $350 \mathrm{msec}$ caused a significant decrease in probability from that of the control condition $[t(13)=2.73$, $p<.025$ ], and this only occurred in males [comparing males to females for the difference between the control and LT350 conditions, $t(12)=2.18, p<.05$ ]. Female response probability was not inhibited at any lead time.

The correlation between response amplitude and response latency was negative in all cases, and was marginally significant in the control condition $(r=-.513$, with the critical value for $p<.05$ being $r=-.531$ ), not significant at lead times of 0,25 , and $50 \mathrm{msec}$, and significant (at $p<.05$ ) at lead times of 75 to $400 \mathrm{msec}$ $(r$ ranged from -.550 to -.760 ). At lead times of $75 \mathrm{msec}$ or greater, larger responses also tended to be faster responses, and this relationship was not as pronounced at lead times less than $75 \mathrm{msec}$. Correlations between response amplitude and probability, and between response latency and probability, were not significant in any condition.

\section{Discussion}

The present study confirms Blumenthal and Gescheider's (1987) conclusion that modification of the human acoustic startle reflex varies as a function of the lead time of a vibrotactile prepulse. The prepulse facilitated response amplitude at a lead time of $25 \mathrm{msec}$ and response latency at lead times of $25,50,75,100,200$, and $350 \mathrm{msec}$. At longer lead times (150 to $350 \mathrm{msec}$ ), the prepulse inhibited response amplitude, but it did not reliably affect response probability. A prepulse at a lead time of 25 msec caused maximum facilitation of the star-

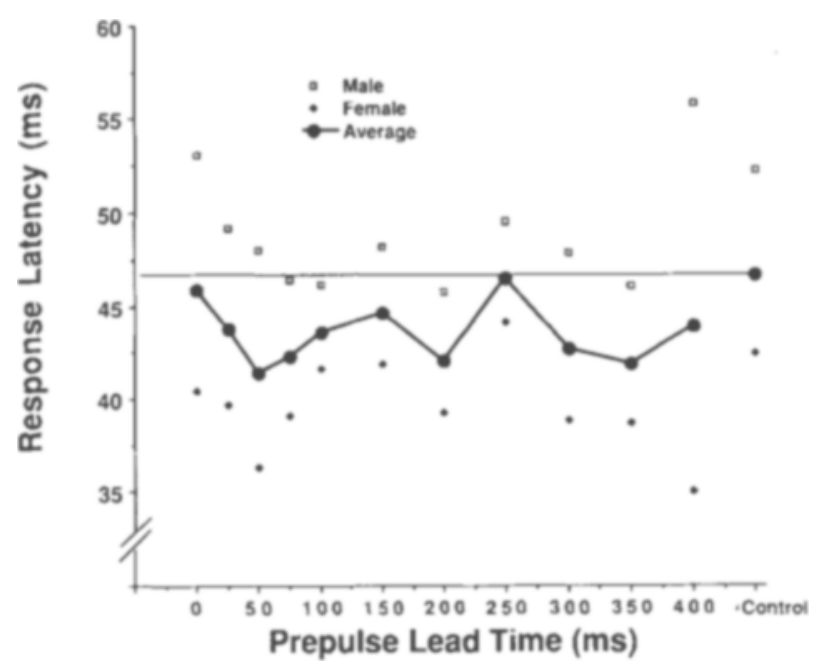

Figure 2. Acoustic startle response latency as a function of vibrotactile prepulse lead time.

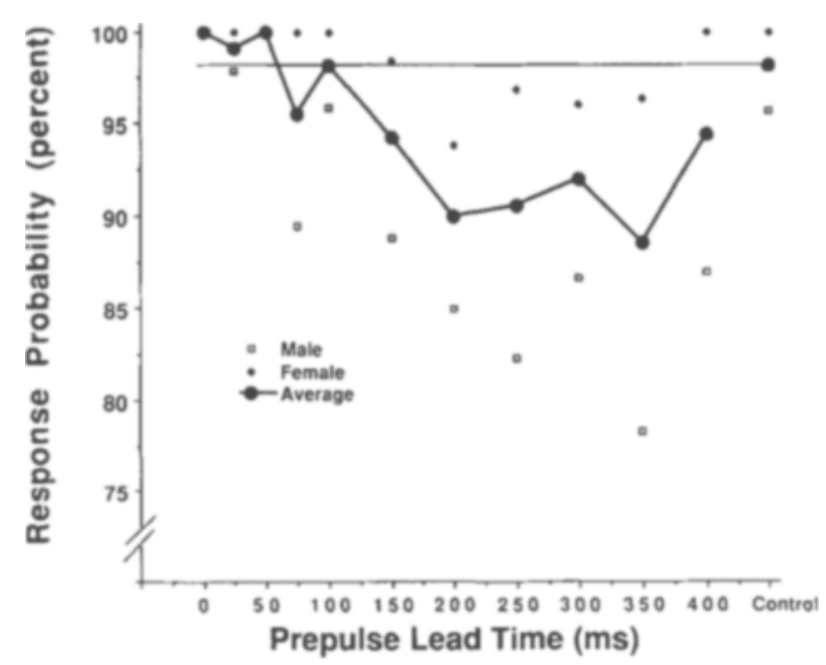

Figure 3. Acoustic startle response probability as a function of vibrotactile prepulse lead time. 
tle response (i.e., increased response amplitude and decreased response latency). Since response amplitude facilitation has never been demonstrated in human beings when a discrete acoustic prepulse at lead times below $100 \mathrm{msec}$ has been used, the fact that the vibrotactile prepulse caused an increase in response amplitude indicates that there is a fundamental difference between the effect of a vibrotactile prepulse and the effect of an acoustic prepulse. Graham (1980) found that the effect of a prepulse on human startle response latency is similar (i.e., there is a decrease in latency) no matter what the modality of the prepulse (acoustic, visual, or electrocutaneous). The vibrotactile prepulses in the present study appeared to increase the efficiency of the acoustic startle stimulus, yielding larger and faster responses. A similar form of crossmodal priming, with a stimulus in one modality facilitating the processing of a startle stimulus in a different modality, has been found by Anthony and Graham (1983, 1985) and by Hackley and Graham (1983).

At a lead time of $200 \mathrm{msec}$, response amplitude was reduced (i.e., there was inhibition) compared to that in the control condition, and response latency was also reduced (there was facilitation), suggesting that two independent neural mechanisms may be at work. The inhibitory and facilitory effects found for response amplitude are generally not present for response probability. Because of a ceiling effect, a significant effect on probability may not be easy to detect (Blumenthal \& Berg, 1986b; Blumenthal \& Gescheider, 1987). Since the probability of responding was so high for the control condition, and since there is a maximum level that response probability can achieve $(100 \%)$, it was very difficult to facilitate a response probability comparable to that in the control condition. Also, if the startle stimuli are normally so effective that response probability is maximal, the presence of a prepulse may not decrease this effectiveness enough for inhibition to appear. This problem could be circumvented with less effective startle stimuli, thereby reducing probability in the control condition and allowing potential inhibitory or facilitory effects to appear (Blumenthal, 1988).

A sex $\times$ lead time interaction was found for response latency, due to lower latency in females than in males for very short or very long lead time conditions. Also, a sex effect for response probability indicates that male response probability was generally lower than female response probability. No effect of sex on response amplitude was found. Blumenthal and Gescheider (1987) found similar sex effects, with females having lower response latency and higher response probability than males.

\section{EXPERIMENT 2}

A second experiment was performed to investigate the effect of presenting two vibrotactile prepulses on the same trial. For the present study, lead times of 25 and $200 \mathrm{msec}$ were chosen, because both had a clear effect on response amplitude (facilitation at 25 -msec lead time and inhibi- tion at 200-msec lead time) and response latency (facilitation at both 25- and 200-msec lead time) in Experiment 1 . It was predicted that the 25 -msec lead time prepulse would increase response amplitude and decrease response latency, and that a 200 -msec lead time prepulse would decrease both response amplitude and response latency. Presentation of both prepulses on the same trial was expected to have an intermediate effect on response amplitude, and to decrease response latency.

\section{Method}

Subjects. Thirteen college undergraduates $(8$ males and $5 \mathrm{fe}$ males), with an average age of 20 years and 11 months (range = 18 years, 2 months to 22 years, 6 months) participated in the experiment. The subjects were selected in the same way as in Experiment 1.

Apparatus and Procedure. The apparatus and procedure were the same as in Experiment 1, with a few exceptions. The stimuli for the second experiment were the same as those in Experiment 1 with respect to intensity, duration, bandwidth, and rise/fall time. The only difference was that the prepulse was delivered at a lead time of 25 or $200 \mathrm{msec}$, or that two prepulses were delivered during the same trial at lead times of 25 and $200 \mathrm{msec}$. There was also a control condition in which no prepulse was delivered. The order of stimulus presentation was determined by presenting blocks of 4 trials each, with each trial block including (in random order) one of each of four stimulus conditions: control (with no prepulse), single prepulse at 25 -msec lead time (LT25), single prepulse at 200-msec time (LT200), two prepulses (one at 25-msec lead time, one at 200msec lead time) (LT25/200), for a total of 40 trials (10 at each condition). Contrasts in a MANOVA were used to compare each trial condition (LT25, LT200, LT25/200) with the control, and to compare each trial condition to each of the other trial conditions. Subject sex was included as a between-subjects factor in these analyses.

\section{Results}

There was no significant difference between males and females in response amplitude, which was similar to the finding in Experiment 1, so the results for the two groups were pooled. The LT25 condition resulted in larger responses than those in the control condition $[F(1,11)=$ $9.69, p<.01]$, the LT200 condition $[F(1,11)=17.93$, $p<.005]$, and the LT25/200 condition $[F(1,11)=9.48$, $p<.025$; see Figure 4]. Responses were also smaller in the LT200 condition than in the LT25/200 condition $[F(1,11)=5.82, p<.05]$. There was no significant difference between the control condition and either the LT200 condition or the LT25/200 condition. The difference from control was less pronounced when both prepulses were presented than when the LT25 prepulse was presented alone $[F(1,11)=9.48, p<.01]$, suggesting that the presence of the 200 -msec lead time prepulse attenuated the facilitory effect of the 25 -msec lead time prepulse. Also, the difference from the control was less pronounced when both prepulses were presented than when the LT200 prepulse was presented alone $[F(1,11)$ $=5.82, p<.05$ ], suggesting that, even though the 200 msec lead time prepulse did not result in significant inhibition, there was at least a tendency in that direction.

For response latency, a significant sex effect was found $[F(1,11)=4.94, p<.05]$, illustrated by shorter latency 


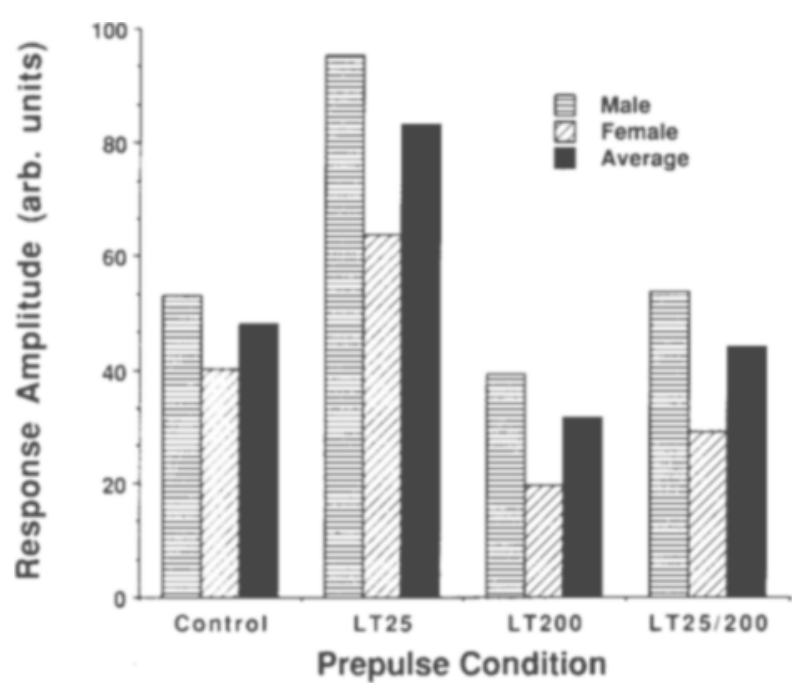

Figure 4. Acoustic startle response amplitude for trials with zero, one, or two vibrotactile prepulses.

for females than for males, which was similar to the finding in Experiment 1 (see Figure 5). This was due to the fact that the LT200 prepulse decreased latency relative to the control condition in females but not in males $[F(1,11)=10.49, p<.01]$. There was no significant sex difference in response latency for the control condition, contrary to the findings in Experiment 1. The LT25 condition resulted in faster responding than in the control condition $[F(1,11)=4.86, p<.05]$, but the condition with both prepulses was not significantly different from the control $[F(1,11)=3.60, p<.085]$.

There was no significant difference between males and females in response probability, which was similar to the finding in Experiment 1 at lead times of 25 and $200 \mathrm{msec}$, so the results of the two groups were pooled. Probability in the LT200 prepulse condition was significantly lower than that in the control condition $[F(1,11)=7.87$, $p<.025]$ and that in the LT25 prepulse condition $[F(1,11)=10.35, p<.01$; see Figure 6]. Neither the LT25 condition nor the LT25/200 condition differed significantly from the control condition, nor did these differ significantly from each other. The response probability was marginally, but not significantly, greater for the LT25 condition than for the LT25/200 condition $[F(1,11)=$ $4.73, p<.053$ ]. Since probability was inhibited by the LT200 prepulse when the prepulse was presented alone, but not when it was accompanied by the LT25 prepulse, it may be the case that the short lead time prepulse actually had a slight facilitory effect on response probability, as was indicated by its ability to partially compensate for the inhibition caused by the long lead time prepulse.

\section{Discussion}

Presenting two prepulses in the same trial caused a combining of the effects of the individual prepulses for response amplitude and probability, but there was no significant effect on response latency. The 25 -msec lead time prepulse caused an increase in response amplitude when presented alone, and this increase was attenuated by the presence of a $200-\mathrm{msec}$ lead time prepulse on the same trial. The single 200-msec lead time prepulse did not result in significant inhibition of response amplitude, failing to replicate the results of Experiment 1, and also failing to replicate the findings of Blumenthal and Gescheider (1987), although there was a slight trend toward inhibition. The reason for this lack of a significant effect is not clear, but it may be due to the presence of a varying number of prepulses on a particular trial. Since multiple prepulses have not been used extensively in human startle modification studies, the reason for this lack of replication is not clear.

Response latency was facilitated by the $200-\mathrm{msec}$ lead time prepulse in females, but not in males, and by the 25 -msec lead time prepulse in both females and males,

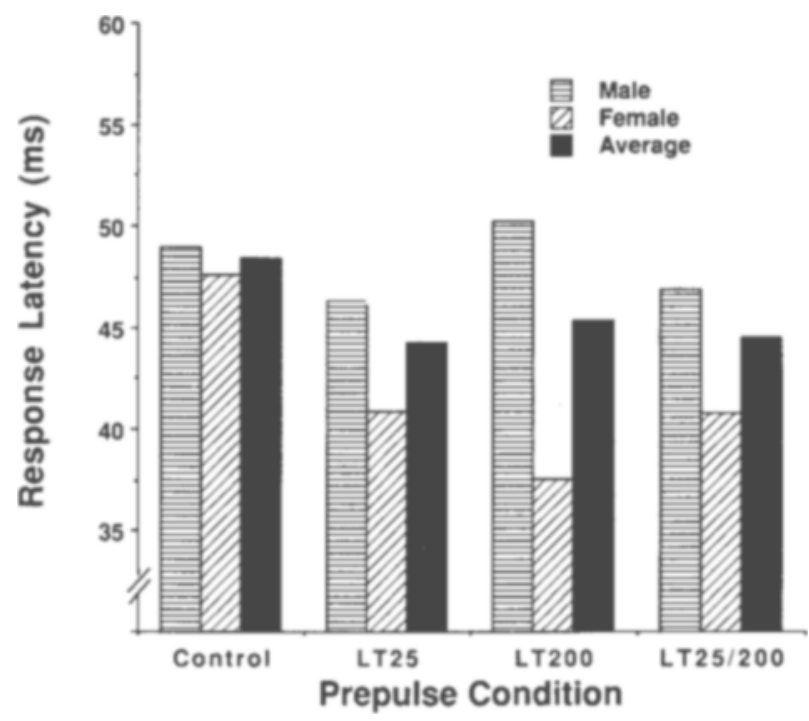

Figure 5. Acoustic startle response latency for trials with zero, one, or two vibrotactile prepulses.

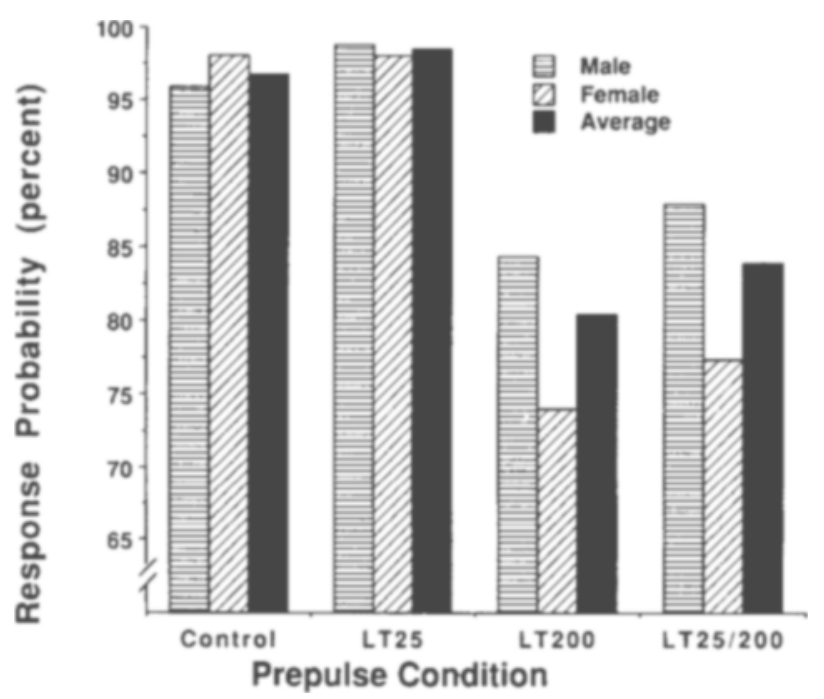

Figure 6. Acoustic startle response probability for trials with zero, one, or two vibrotactile prepulses. 
in support of the findings in Experiment 1. Presenting two prepulses on the same trial did not significantly affect response latency. The interaction between subject sex and prepulse lead time for this measure confuses this lack of replication, since the latency effects shown in Figure 5 are more pronounced for females than for males. What these sex differences suggest is that there may be genuine difference in response latency between males and females in studies involving vibrotactile prepulses, with female latency being shorter (since similar effects were also found by Blumenthal \& Gescheider, 1987, who used prepulses similar to those in the present study). However, these sex differences are not reliable at present.

Response probability was inhibited by the 200 -msec lead time prepulse, but no effect of the 25 -msec lead time prepulse was found. These findings partially support those in Experiment 1, where the 25-msec lead time prepulse had no effect on probability (possibly due to a ceiling effect), and the 200 -msec lead time prepulse also had no significant effect on response probability-although the data suggest a slight but not significant trend toward inhibition $[F(1,12)=3.25, p<.097]$.

As in Experiment 1 , a sex $\times$ lead time interaction was found for response latency, and no effect of sex on response amplitude appeared. The effect of sex on response probability found in Experiment 1 was not found in Experiment 2, perhaps because of the difference in the number of lead time conditions used in the two studies. While these sex differences may be spurious, they may also be genuine, caused by variations in sensitivity to stimuli in females as a function of their menstrual cycle (Davis \& Ahroon, 1982; Gescheider, Verrillo, McCann, \& Aldrich, 1984). There were no significant sex differences in vibrotactile threshold, but response latency in the control condition was lower for females than for males (response amplitude and probability showed no significant sex differences in the control condition). These sex differences appear to involve greater sensitivity to the acoustic startle stimulus in females than in males, independent of the vibrotactile prepulse (although this greater sensitivity is specific to the response latency measure). In fact, the amount of inhibition or facilitation caused by the prepulse was generally not significantly different for the two sexes. Information regarding position in the menstrual cycle was not collected in the present study, which makes these sex effects difficult to interpret. We recommend that researchers who use vibrotactile prepulses in startle modification studies collect information regarding the menstural cycle of their female subjects, if possible, since this information may be useful for the explanation of this unexpected influence of sex on reflex elicitation and modification.

\section{GENERAL DISCUSSION}

The current experiments involved an attempt to evaluate a crossmodal interaction that has different effects on the startle reflex, depending on the lead time at which the prepulse is presented. The findings clearly show that the human acoustic startle reflex can be modified by a weak vibrotactile prepulse. The effect of a vibrotactile prepulse is different from the effect of an acoustic prepulse, because the vibrotactile prepulse causes an increase in amplitude at short lead times, while this effect has not been demonstrated with an acoustic prepulse. At longer lead times, a vibrotactile prepulse generally causes a reduction of response amplitude, an effect that is also found with acoustic and visual prepulses (Graham, 1980). A prepulse at a short lead time causes a decrease in response latency, and this effect is similar across modalities (Graham, 1980). Using pigeons, Stitt, Hoffman, Marsh, and Schwartz (1976) have shown that latency facilitation requires a more intense prepulse than does amplitude inhibition.

A vibrotactile prepulse at a $25-\mathrm{msec}$ lead time caused a significant increase in acoustic startle response amplitude. Given the conduction time for tactile input from the hand to the midbrain of $15-20 \mathrm{msec}$ in human beings (Desmedt \& Cheron, 1982), the tactile and acoustic activation may overlap in this condition. Gescheider, Kane, Sager, and Ruffolo (1974) provide evidence that suggests that auditory stimulation may facilitate neural activity in the vibrotactile system, when acoustic and tactile stimuli are presented simultaneously to human subjects. Response facilitation by a stimulus presented simultaneously with the startle stimulus is a well-established finding with respect to human beings (Hoffman et al., 1981). Similar crossmodal facilitation was clearly seen at short lead times in the present study, and this type of facilitation may be difficult to see if the prepulse and startle pulse are in the same modality, due to the fact that the startle pulse could mask the prepulse, preventing the prepulse from being effective. In order to see if this facilitation by a simultaneous prepulse is due to peripheral or central processes, the prepulse and startle stimulus could be presented in the same modality but activate spatially separate receptors (i.e., different critical bands in the acoustic system, different receptor types in the tactile system, different wavelengths in the visual system).

When two prepulses were presented on the same trial, the inhibition of response probability caused by the long lead time prepulse was attenuated by the presence of the short lead time prepulse. For response amplitude, the facilitation caused by a short lead time prepulse was attenuated by the presence of the long lead time prepulse. The facilitation caused by the short lead time prepulse may be less pronounced if the response to the startle stimulus has already been inhibited by the long lead time prepulse, illustrating an interaction of the inhibition and facilitation effects produced by the two prepulses on a single trial.

Using acoustic prepulses and measuring acoustic startle in rats, Stitt, Hoffman, and Marsh (1976) suggest that startle reflex amplitude inhibition and latency facilitation are mediated by separate neural mechanisms. Graham and Murray (1977) speculate that these mechanisms may be based on the transient and sustained neurons described 
by Gersuni (1971). The present studies support this hypothesis, and show that startle amplitude and latency can be modified in different ways by crossmodal stimulation. Knowledge of the interactions between sensory modalities can aid in the determination of normal and potentially deficient stimulus processing, and the startle modification paradigm provides a noninvasive way to assess perceptual functions, and to thereby infer the mechanisms underlying stimulus processing.

\section{REFERENCES}

ANTHONY, B. J. (1985). In the blink of an eye: Implications of reflex modification for information processing. In P. K. Ackles, J. R. Jennings, \& M. G. H. Coles (Eds.), Advances in psychophysiology: Vol. I (pp. 167-218). Greenwich, CT: JAI Press.

Anthony, B. J., Graham, F. K. (1983). Evidence for sensoryselective set in young infants. Science, 220, 742-744.

Anthony, B. J., \& Graham, F. K. (1985). Blink reflex modification by selective attention: Evidence for the modulation of 'automatic' processing. Biological Psychology, 20, 43-59.

BÉKÉsy, G. von (1974). A new audiometer. Acta Otolaryngologica, $35,411-422$.

Blumenthal, T. D. (1988). The startle response to acoustic stimuli near startle threshold: Effects of stimulus rise and fall time, duration, and intensity. Psychophysiology, 25, 607-611.

BlumenthaL, T. D., BerG, W. K. (1986a). The startle response as an indicator of temporal summation. Perception \& Psychophysics, 40, 62-68.

Blumenthal, T. D., \& BerG, W. K. (1986b). Stimulus rise time, intensity, and bandwidth effects on acoustic startle amplitude and probability. Psychophysiology, 23, 635-641.

Blumenthal, T. D., Gescheider, G. A. (1987). Modification of the acoustic startle reflex by a tactile prepulse: The effects of stimulus onset asynchrony and prepulse intensity. Psychophysiology, 24, 320-327.

Blumenthal, T. D., \& Verma, A. (1988, April). The effects of low levels of caffeine on the human startle reflex. Paper presented at the Southeastern Psychological Association Convention, New Orleans.

DAvis, M. J., AHROON, W. A. (1982). Fluctuations in susceptibility to noise-induced temporary threshold shift as influenced by the menstrual cycle. Journal of Auditory Research, 22, 173-187.

Desmedt, J. E., \& Cheron, G. (1982). Somato-sensory evoked potentials in man: Subcortical and cortical components and their neural basis. Annals of the New York Academy of Sciences, 388, 388-400.

Gallager, D. W., Kehne, J. H., Wakeman, E. A., Davis, M. (1983). Developmental changes in pharmacological responsivity of the acoustic startle reflex: Effects of picrotoxin. Psychophysiology, 79, 87-93.

Gersuni, G. V. (1971). Temporal organization of the auditory function. In G. V. Gersuni (Ed.), Sensory processes at the neuronal and behavioral levels (pp. 85-114). New York: Academic Press.

Gescheider, G. A., Kane, M. J., SAger, L. C., Rufrolo, L. J. (1974). The effect of auditory stimulation on responses to tactile stimuli. Bulletin of the Psychonomic Society, 3, 204-206.

Gescheider, G. A., Verrillo, R. T., McCann, J. T., Aldrich, E. M. (1984). Effects of the menstrual cycle on vibrotactile sensitivity. Perception \& Psychophysics, 36, 586-592.

Graham, F. K. (1975). The more or less startling effects of weak prestimulation. Psychophysiology, 12, 238-248.

Graham, F. K. (1980). Control of reflex blink excitability. In R. F. Thompson, L. H. Hicks, \& V. B. Shvyrkov (Eds.), Neural mechan- isms of goal-directed behavior and leaming (pp. 511-519). New York: Academic Press.

Graham, F. K., \& Murray, G. M. (1977). Discordant effects of weak prestimulation on magnitude and latency of the reflex blink. Physiological Psychology, 5, 108-114.

HackleY, S. A., \& Graham, F. K. (1983). Early selective attention effects on cutaneous and acoustic blink reflexes. Physiological Psychology, 11, 235-242.

Hoffman, H. S., Cohen, M. E., \& English, L. M. (1985). Reflex modification by acoustic signals in newborn infants and in adults. Journal of Experimental Child Psychology, 39, 562-579.

Hofman, H. S., Cohen, M. E., \& STTTT, C. L. (1981). Acoustic augmentation and inhibition of the human eyeblink. Journal of Experimental Psychology: Human Perception \& Performance, 7, 1357-1362.

Hoffman, H. S., \& Ison, J. R. (1980). Reflex modification in the domain of startle: 1 . Some empirical findings and their implication for how the nervous system processes sensory input. Psychological Review, 87, 175-189.

Ison, J. R., Foss, J. A., Falcone, P., Sakovitz, L., Adelson, A. A., \& Burton, R. I. (1986). Reflex modification: A method for assessing cutaneous dysfunction. Perception \& Psychophysics, 40, 164-170.

Ison, J. R., HAMmoND, G. R. (1971). Modification of the startle reflex in the rat by changes in the auditory and visual environment. Journal of Compararive \& Physiological Psychology, 75, 435-452.

Leitner, D. S., Powers, A. S., Hoffman, H. S. (1979). The neural system for the inhibition of startle. Bulletin of the Psychonomic Society, 14, 410-412.

Marsh, R., Hoffman, H. S., Stitt, C. L. (1978). Reflex inhibition audiometry: A new objective procedure. Acta Otolaryngologica, 85, 336-341.

Ornitz, E. M., Guthrie, D., Kaplan, A. R., Lane, S. J., NorMAN, R. J. (1986). Maturation of startle modulation. Psychophysiology, 23, 624-634.

ReITER, L. A., Ison, J. R. (1977). Inhibition of the human eyeblink reflex: An evaluation of the sensitivity of the Wendt-Yerkes method for threshold detection. Journal of Experimental Psychology: Human Perception \& Performance, 3, 325-336.

Stitt, C. L., Hoffman, H. S., MARSH, R. R. (1976). Interaction versus independence of the startle-modification processes in the rat. Journal of Experimental Psychology: Animal Behavior Processes, 2 , 260-265.

Stitt, C. L., Hoffman, H. S., Marsh, R. R., Schwartz, G. M. (1976). Modification of the pigeon's visual startle reaction by the sensory environment. Joumal of Comparative \& Physiological Psychology, 90, 601-619.

\section{NOTES}

1. Some researchers in this area report average response magnitude, while others report average response amplitude. In most cases, response magnitude is the product of response amplitude and response probability. The distinction between response magnitude and amplitude becomes important as response probability decreases. See Blumenthal and Berg (1986b) for a description of these differences in response measurement.

2. For readers who refer to Graham (1980), note that the $y$-axis of Figure 35.2 (p. 514) is mislabeled. Since facilitation of latency involves a decrease below control levels, the $y$-axis should be labeled "Percent Facilitation." As it is presently labeled, this graph suggests that latency increased in the presence of a prepulse, when it actually decreased. Alternatively, the functions for latency data could be inverted, making the current $y$-axis correct.

(Manuscript received August 17, 1988; revision accepted for publication March 2, 1989.) 